\title{
Numerical simulation of miniature antennas applicators of microwave radiometry for diagnostics of the functional state of the brain
}

\author{
Vitaly Leushin ${ }^{1, *}$, Sergey Chizhikov $^{1}$, Sergey Vesnin ${ }^{1,2}$, Mikhail Sedankin ${ }^{1,3}$, \\ Igor Porokhov ${ }^{4}$, Dmitry Gorbachev ${ }^{1,5}$, and Evgenia Gorlacheva ${ }^{6}$ \\ ${ }^{1}$ Bauman Moscow State Technical University, Research Institute of Radioelectronics and laser \\ technology, 105005, 2-ya Baumanskaya st., 5, Moscow, Russia \\ ${ }^{2}$ LLC "RTM Diagnostics”, Russia, Technical department, 105082, Pochtovaya B. str., 55/59, \\ Moscow, Russia, \\ ${ }^{3}$ State Research Center - Burnasyan Federal Medical Biophysical Center of Federal Medical \\ Biological Agency, Russia, Department of radiation epidemiology, 123098, Marshala Novikova str. \\ 23, Moscow, Russia \\ ${ }^{4}$ JSC "CNIRTI named after academician A.I. Berg", Antennas department, 107078, Novaya \\ Basmannaya, 20/8, Moscow, Russia \\ ${ }^{5}$ JSC Radio Engineering Corporation "VEGA", Departmenf of Radar Systems, 121170, Kutuzovsky \\ prospekt, 34, Moscow, Russia \\ ${ }^{6}$ Bauman Moscow State Technical University, Department of industrial logistic, 105005, 2-ya \\ Baumanskaya st., 5, Moscow, Russia
}

\begin{abstract}
This paper describes the use of Finite Difference Time Domain technique of numerical modeling for development and simulation symmetrical dipole with triangular shoulders placed in a cylindrical housing and a spiral antenna placed in a cylindrical housing for use in microwave radiometry. The new sensors have been tested and validated on different phantoms and biological tissues. Results suggest sufficient characteristics of broadband antennas for potential use in brain functional diagnostics.
\end{abstract}

\section{Introduction}

It is known that there is a connection between the violation of the functioning of organs and tissues of the body and changes in their local temperature. The great interest of scientists in the fields of medicine, biology and biophysics to microwave radiometry is due to the fact that it is a non-invasive method of measuring the internal temperatures of the human body based on the registration of their own microwave radiation. Reception of radiation is carried out through the use of special medical antennas and highly sensitive receivers radiometers. To determine the temperature of internal human tissues, it is necessary to accept the energy of thermal radiation in the microwave range. An overview of the use of microwave radiometry in medicine is presented in [1]. Because of the small values of noise signals taken from a biological object, it is very important to protect against various

* Corresponding author: ra3bu@yandex.ru 
electromagnetic signals, because the level of interference can be so large that it is difficult to assess the background of their informative signal. Therefore, one of the main requirements for antennas, in addition to wide-band in the microwave range and miniaturization, is their noise immunity. In [2] the results of modeling of a number of printed antennas are presented, which confirmed the possibility of their use in multichannel radiometers. Currently, the following main types of medical antennas for microwave radiometry are the most widely used: waveguide, printed, vibratory or frame antennas, as well as intracavitary antennas [3-6]. Unlike waveguide antennas, which have a waveguide segment between the emitter and the antenna aperture, printed antennas have the emitter printed directly on the dielectric substrate. Substrate thickness normally does not exceed 2 $\mathrm{mm}$. Therefore, the printed antennas are lighter than waveguides antennas, cheaper, have a small height and has less impact on the temperature of the skin. In recent years, these antennas have become widespread. In many cases, printed antennas are used in conjunction with a bolus. A bolus is a thin dielectric layer that is placed between an antenna and a biological object. Bolus allows you to align the antenna and increase the distance between the emitter and the biological object to the reactive fields arising in the emitter, do not fall into the biological object. This allows you to increase the depth of measurement. Bolus sizes are usually slightly larger than or equal to the size of the antenna aperture. The following classes of printed antennas can be distinguished: spiral antennas [7-9], elliptical antennas[10], patch antennas [11,12], slot antennas [13-18] and dipole or vibrator printed antennas[19]. For fig.1 the main types of existing antennas are presented. The excitation system is connected in the center of the spiral or in the place indicated by a circle (fig. $1 \mathrm{a})$. We formulated the criteria for optimum selection of antenna in concordance with technical requirements: high rates of measurement depth of the antenna because of power decrease accepted from skin layers of the patient; high level of noise shielding; miniature sizes of antenna; sufficient matching of antenna with biological tissues during the operation.

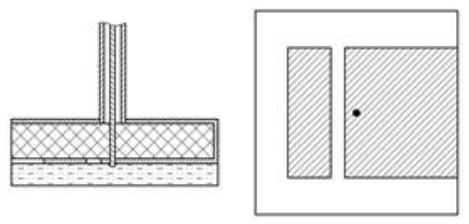

a

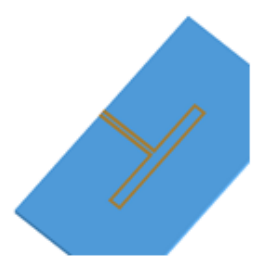

d

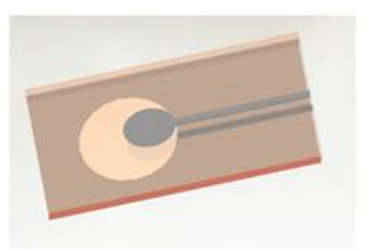

b
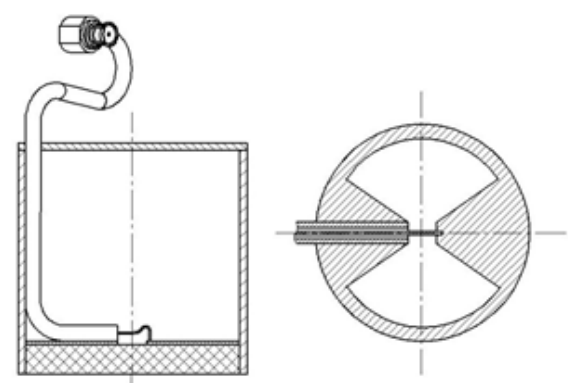

e

Fig. 1. Main types of medical printed antennas: a - patch antenna; b - elliptical antenna; $\mathrm{c}$ - spiral antenna; $\mathrm{d}$ - dipole printed antenna; $\mathrm{e}$ - slot antenna.

It is necessary to minimize longitudinal component of electric field at the limit of the biological object's division. Many printed antennas have significant longitudinal component of electric field. Such antennas are worse than waveguide antennas in the 
measurement depth. Widespread dipole (vibrator) antennas have acceptable sizes and low noise immunity therefore can't be applied in medicine without a special room shielding. The purpose of this work is to consider the possibility of creating new types of noiseprotected antennas for measuring brain temperatures by microwave radiometry. Below are the results of modeling and analysis of the main characteristics of two printed antennas: vibratory and spiral antennas.

\section{Main part}

The microwave antenna measures an equivalent brightness temperature $T_{b r}$ determined from the weighted volumetric average temperature within the sense region of the antenna. The power collected by the antenna $(\mathrm{P})$ is directly proportional to $\mathrm{T}_{\mathrm{br}}$ and is given by:

$$
P=k T_{b r} \Delta f
$$

where $\mathrm{k}=1,38 \cdot 10^{-23} \mathrm{~J} /{ }^{\circ} \mathrm{K}$ is the Boltzmann constant; $\Delta \mathrm{f}$ - working band of radiometer, GHz.

The brightness temperature, measured by a medical radiometer, is related to the physical temperature $\mathrm{T}(\mathrm{r})$ in the human tissue located at position $\mathrm{r}$ within a sense volume $\mathrm{V}$, and $\mathrm{S}_{11}$ as the power reflection coefficient due to mismatch at the antenna/biological tissues, the temperature measured by the radiometer is given:

$$
T_{b r}=\left(1-S_{11}^{2}\right) \int_{-\infty}^{\infty} T(r) \cdot W(r) d V
$$

where $\mathrm{W}(\mathrm{r})$ is the weight radiometric function.

The most of radiometers use special circuits, which compensate influence of reflection coefficient on outcomes measurement, and so $\mathrm{S}_{11}=0$ is usually accepted in calculations. Thus $\mathrm{T}_{\text {br }}$ measured by radiometer is defined as:

$$
T_{b r}=\int_{-\infty}^{\infty} T(r) W(r) d V
$$

$\mathrm{W}(\mathrm{r})$ weighting radiometric function of antenna is defined as:

$$
W(r)=\frac{\sigma(r)|E(r)|^{2}}{\int_{-\infty}^{\infty} \sigma(r)|E(r)|^{2} d V}
$$

where E(r) - electric field intensity of lossy medium created by antenna in volume of biological object when in the transmission mode, $\sigma(\mathrm{r})$ - tissues electrical conductivity of biological tissues.

$\mathrm{W}(\mathrm{r})$ depend on $\mathrm{E}(\mathrm{r})$ is the electric field intensity of the antenna and $\sigma(\mathrm{r})$ is the electrical conductivity of the tissues. To develop an antenna for brain diagnostics, it is necessary to calculate its electric field E (r) in the near field zone. Numerical methods used in the program of electrodynamic modeling were used to calculate the electric field. The program numerically solves the Maxwell equations by the technique FDTD [20]. The FDTD technique permits the analysis of interactions of electromagnetic waves with material bodies of any desired shape. An FDTD code that can be used for calculating the scattering parameters as well as the current and field distribution over the surface and edges is generated. First, select the counting area (the volume in which the calculation is performed), 
the grid resolution (calculation step), and the boundary conditions. Inside the counting domain, the model of the body under study with certain electrophysical properties is located. The program numerically solves the Maxwell equations in finite-difference form, and the values of the field components at each new step are found from the values at the previous steps. To select the optimal antenna design that meets the necessary requirements for their application in radiometry, was simulated near-field vibratory and helical antenna. Antenna placed in the insulating housing with a wall thickness of $2 \mathrm{~mm}$. The substrate is a printed circuit antenna made from fluoroplastic and have a diameter of $26 \mathrm{~mm}$. Caps of housings are also made of fluoroplastic and have a thickness of $2 \mathrm{~mm}$. For the analysis of vibratory antenna was chosen symmetrical dipole with triangular shoulders. The size of the projection of the current-carrying elements of the antenna module on the surface of the biological load is $20 \times 10 \mathrm{~mm}$. the 3D model of the antenna with triangular arms is shown in Fig. 2 a.

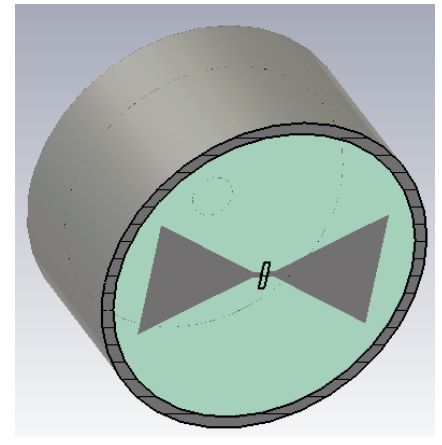

a

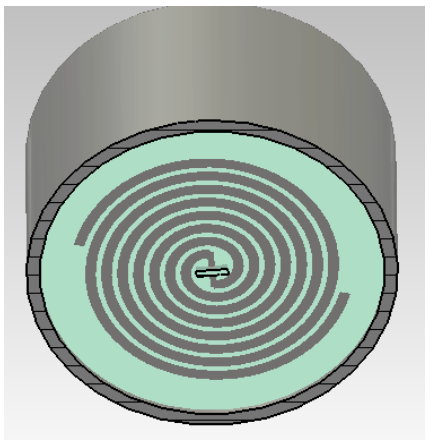

b

Fig. 2. 3D model of a symmetrical dipole with triangular shoulders placed in a cylindrical housing (a); 3D model of a spiral antenna placed in a cylindrical housing (b).

As a model of a spiral antenna was selected arithmetic of the double helix: the width of the conductors of the spiral branches of $1.4 \mathrm{~mm}$, the gap between adjacent turns of $1 \mathrm{~mm}$. 3D model of the antenna with the arithmetic of the double helical structure shown in Fig.2 b. The antenna's design is shown in Fig 2. The All the layers of the model are isotropic in physical properties. The program automatically creates a grid model, and the grid spacing is uneven for different subdomains. Conducting surfaces are treated by setting the tangential electric field components to zero. The study frequency range was 3.2-4.4 GHz. Antenna is in direct contact with the biological load was simulated. Simulator biological load was a box the size of 160x160x60 mm with appropriate electrical properties in muscle and bone tissue (tab. 1), obtained on the basis of [21].

Table 1. Averaged electrophysical parameters of biological tissues of the human head

\begin{tabular}{|l|l|l|l|}
\hline $\begin{array}{l}\text { Biological } \\
\text { tissues }\end{array}$ & $\begin{array}{l}\text { Dielectric } \\
\text { constant, }\end{array}$ & $\begin{array}{l}\text { Electrical } \\
\text { conductivity, Sm/m }\end{array}$ & $\begin{array}{l}\text { Biological layer } \\
\text { thickness, mm }\end{array}$ \\
\hline Skin & 36,92 & 2,01 & 2 \\
\hline $\begin{array}{l}\text { Fat and } \\
\text { bone }\end{array}$ & 5,17 & 0,156 & 4 \\
\hline Brain & 46,76 & 2,93 & 89 \\
\hline
\end{tabular}

\section{Results}

The first most critical characteristic of an antenna is its macthing with biological tissues. Varying the geometrical parameters of antennas, you can achieve the best VSWR. Besides 
efficiency, the band is chosen based on the constraint VSWR $<2$ in order to minimize mismatch between the antenna and tissue. Analysis of the frequency responces shown in Fig. 3 and 4 showed that the VSWR is optimal.

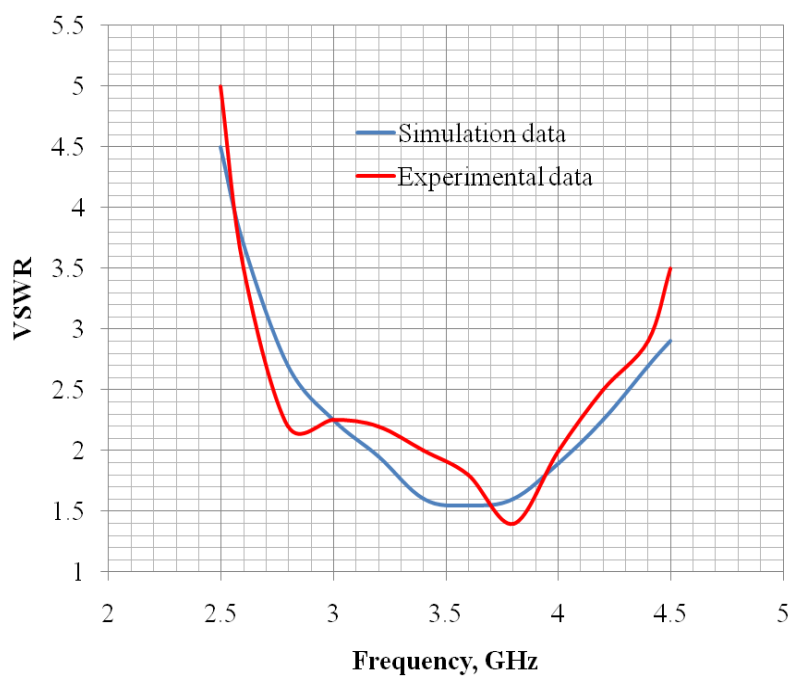

Fig. 3. Frequency responce of VSWR of the symmetric dipole with triangular shoulders: simulation and experimental data.

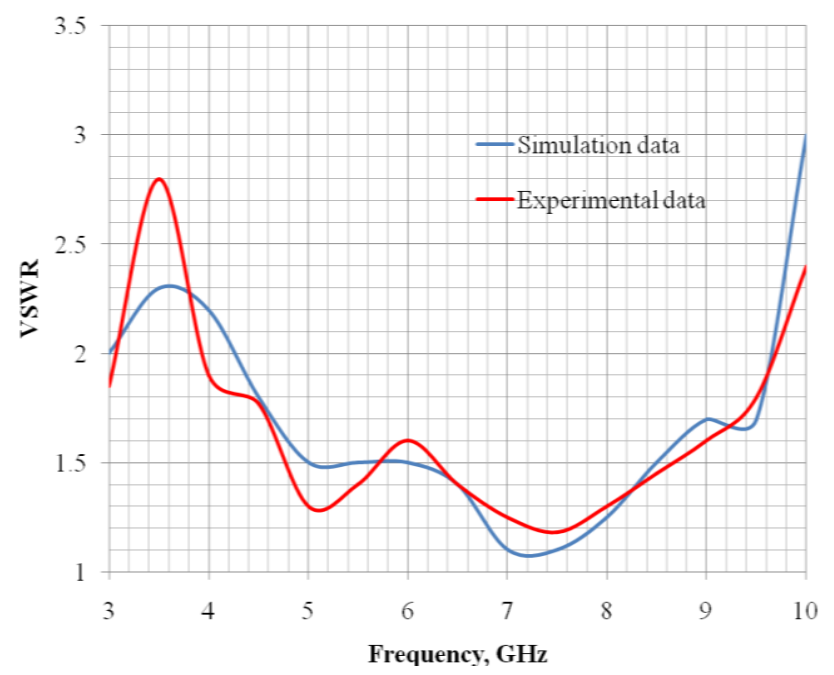

Fig. 4. Frequency responce of spiral antenna VSWR: simulation and experimental data.

\section{Conclusion}

Analysis of the results of calculations of the frequency responses of VSWR of the antennas showed that the considered broadband antennas applicators has good frequency response and electrical performance and are promising for their use in the composition of radiothermography to measure the brightness temperature in the depths of the human body including in the diagnosis of the functional state of the brain.

Research was supported by a grant of the Russian Science Foundation (project No. 19-19-00349). 


\section{References}

1. S.G. Vesnin et al., Research of a microwave radiometer for monitoring of internal temperature of biological tissues. Eastern-European Journal of Enterprise Technologies, v. 4, N. 5 (100). pp.6-15 (2019)

2. A.F. Bobrikhin, A.G. Gudkov, V.Yu. Leushin, V.F. Los, I.O. Porokhov, I.A. Sidorov, Modeling of the dipole, helical and cavity-slot antennas applicators for multichannel medical radiothermographs. CriMiCo 2014 - 24th International Crimean Conference Microwave and Telecommunication Technology, Sevastopol, 7-13 Sept. 2014, pp. 2014-1246 (2014)

3. M.K. Sedankin, V.Yu. Leushin, A.G. Gudkov, S.G. Vesnin, I.A. Sidorov, S.V. Agasieva, L.M. Ovchinnikov, N.A Vetrova, Antenna applicators for medical microwave radiometers, Biomedical Engineering, v. 52, No. 4, pp. 235-238 (2018)

4. D.N. Chupina, M.K. Sedankin, S.G. Vesnin, Application of modern technologies of mathematical simulation for the development of medical equipment, 11th IEEE International Conference on Application of Information and Communication Technologies, AICT 2017 - Proceedings 11. C. 8687066 (2019)

5. M.K. Sedankin et al., Development of patch textile antenna for medical robots, 2018 International Conference on Actual Problems of Electron Devices Engineering, APEDE 2018 , pp. 413-420 (2018)

6. M.K. Sedankin, V.Yu. Leushin, S.G. Vesnin, I.A. Sidorov, S.V. Agasieva, A.V. Markin, Mathematical simulation of heat transfer processes in a breast with a malignant tumor, Biomedical Engineering, v. 52. No.3. pp. 190-194 (2018)

7. S. Jacobsen, P. R. Stauffer, H.O. Rolfsnes, Characteristics of mircostrip muscle-loaded single-arm archimedean spiral antenna as investigated by FDTD numerical computations, IEEE Transaction on Biomedical Engineering, v. 52, No. 2. pp.321-33 (2005)

8. D.B. Rodrigues et al., Microwave radiometry for noninvasive monitoring of brain temperature, Emerging Electromagnetic Technologies for Brain Diseases Diagnostics, Monitoring and Therapy, Springer, Cham, pp. 87-127 (2018)

9. P.R. Stauffer et al., Stable microwave radiometry system for long term monitoring of deep tissue temperature, Energy-based Treatment of Tissue and Assessment VII. International Society for Optics and Photonics, v. 8584, C. 85840R, (2013)

10. O. Klemetsen, S. Jacobsen, Improved radiometric performance attained by an elliptical microwave antenna with suction, IEEE Transactions on Biomedical Engineering, v. 59(1), pp.263-271 (2012)

11. P. Momenroodaki, Z. Popovic, R. Scheeler, A 1.4-GHz radiometer for internal body temperature measurements, European Microwave Conference (EuMC), IEEE, pp. 694$697(2015)$

12. P. Momenroodaki et al., Noninvasive internal body temperature tracking with nearfield microwave radiometry, IEEE Transactions on Microwave Theory and Techniques, No 5, pp. 2535-2545 (2017)

13. P.Y. Cresson et al., Temperature measurement by microwave radiometry, IEEE International Instrumentation and Measurement Technology Conference, Victoria (Vancouver Island, Canada), pp. 1344 - 1349 (2008)

14. V.M. Ravi, K. Arunachalam, A low noise stable radiometer front-end for passive microwave tissue thermometry, Journal of Electromagnetic Waves and Applications, v. 35, No 6, pp. 743-758 (2019)

15. M.K. Sedankin et. al., Development of a miniature microwave radiothermograph for monitoring the internal brain temperature, Eastern-European Journal of Enterprise Technologies, v. 3(5), pp. 26-36 (2018) 
16. L. Dubois et al., Contact-less sensors for temperature measurement by microwave radiometry in medical or industrial applications, Proceedings of ISAP, Niigata (Japan), pp.1262-1265 (2007)

17. Clarisse Beaucamp-Ricard et al., Temperature measurement by microwave radiometry. IEEE transactions on instrumentation and measurement, v. 58, No 5, pp. 1712-1719 (2009)

18. M. R. Tofighi, Dual-mode planar applicator for simultaneous microwave heating and radiometric sensing, Electronics letters, v. 48, No 20, pp. 1252-1253 (2012)

19. Z. Popovic, P. Momenroodaki, R. Scheeler, Toward wearable wireless thermometers for internal body temperature measurements, IEEE Communications Magazine, No 10, pp. 118-125 (2014)

20. I. Valuev et al., Creating Numerically Efficient FDTD Simulations Using Generic C++ Programming, Lecture Notes in Computer Science (LNCS) 4707, 213 (2007)

21. S. Gabriel, R. W. Lau, C. Gabriel, The dielectric properties of biological tissues, II. Measurements in the frequency range $10 \mathrm{~Hz}$ to $20 \mathrm{GHz}$, .Physics in medicine \& biology, v. 41, No 11, pp. 2251 (1996) 